\title{
Arterial Blood Pressure Measurement and Pulse Wave Analysis
}

\author{
${ }^{1}$ Prof.Subhash k.Ohal, ${ }^{2}$ Prof.R.J.Vaidya \\ ${ }^{1,2}$ M.Tech(E\&TC) Bharati vidyapeeth,pune
}

\begin{abstract}
The most common method of clinical measurement of arterial blood pressure is by means of the cuff sphygmomanometer. This instrument has provided fundamental quantitative information on arterial pressure in individual subjects and in populations and facilitated estimation of cardiovascular risk related to levels of blood pressure obtained from the brachial cuff. Although the measurement is taken in a peripheral limb, the values are generally assumed to reflect the pressure throughout the arterial tree in large conduit arteries. Since the arterial pressure pulse becomes modified as it travels away from the heart towards the periphery, this is generally true for mean and diastolic pressure, but not for systolic pressure, and so pulse pressure. The relationship between central and peripheral pulse pressure depends on propagation characteristics of arteries. Hence, while the sphygmomanometer gives values of two single points on the pressure wave (systolic and diastolic pressure), there is additional information that can be obtained from the time-varying pulse waveform that enables an improved quantification of the systolic load on the heart and other central organs. This topical review will assess techniques of pressure measurement that relate to the use of the cuff sphygmomanometer and to the non-invasive registration and analysis of the peripheral and central arterial pressure waveform.
\end{abstract}

Keywords: Arterial pressure, sphygmomanometer, hypertension, ageing, cardiovascular risk, pulse pressure, heart rate, pulse waveform, pulse wave

\section{Introduction}

Arteriosclerotic disease is the leading cause of death in many developed countries. It affects 10-25\% of people over the age of 55 and it can lead to myocardial infarction and death. It occurs because of increased or decreased blood pressure. Irregular pulse pressure may be a strong predictor of heart problems, especially for aged people. The increased arterial stiffness in the elderly leads to increased wave reflection, which increases central pulse pressure. This accelerates the speed at which the left ventricular ejection pressure wave travels through the arteries, and leads to an earlier return of the reflected pressure wave back to the left ventricle. The reflected pressure wave starts to arrive more and more during systole, where it augments the late systolic on the left ventricle. Because the wave is arriving less during diastole, it reduces the coronary artery perfusion pressure during this critical period. Therefore, increased arterial stiffness leads to a greater risk of angina and heart attack, stroke and heart failure. Arterial pulse wave analysis, studies the wave shape of the blood pressure pulse [2]. The pulse pressure waveform results from variations in blood pressure due to variation in blood flow through the blood vessels during a cardiac cycle. The shape of the pulse pressure waveform is determined by the ejection pattern from the left ventricle, the mechanical properties of the arterial system, and the peripheral vascular resistance. Hence, heart function and artery elasticity are the major determinants creating pulse wave; thus age, gender and other physiological factors directly affects the wave contour. By diagnosing the pulse pressure waveform, various pathological and physiological information of the cardiovascular system can be easily obtained accurately. Abnormal and uncontrolled increase or decrease in pressure results in morbidity and mortality from treatment failures and increased health care costs. Although defining the precise public health risk and estimating the increase in costs is not a simple undertaking, there is little doubt that abrupt variations in pressure is a serious global problem. Most of the clinicians are not able to give the best therapy to patients because there is no such system or method currently available for continuous monitoring of blood pressure wave. So there is a need of developing methods for early detection of abrupt blood pressure changes and internal injury. Conventional techniques in clinical practice now a-days don't provide continuous, long time monitoring and more human errors occur during interpretation of results. So there is a need to develop systems with continuous waveform analysis of pulse pressure to avoid such errors. The aim of this study is to develop a noninvasive[3],strap based[5], reliable system along with feature extraction algorithm[7] for diagnostic purposes and to evaluate various pulse parameters such as reflection index, stiffness index to estimate various arterial properties and onset of hemorrhagic conditions.

\section{Morphology}

A beating heart generates pressure and flow waves which propagate throughout the arterial system. These blood pressure waves serve as a scanner of the arterial tree of the whole body. There are numerous points in the body where these waves can be acquired. But, the most feasible and easily detectable location is the wrist 
where radial artery is situated. The shapes of wrist-pulse waveforms are altered by their continuous interaction with the non uniform arterial system. The pressure waves expand the arterial walls as traveling, and the expansions are palpable as the wrist-pulse. The palpable wrist-pulses consists of one forward traveling wave component, the collective waves running from heart to periphery and contains information of the heart itself; and one backward traveling wave component, the collective waves containing information of the reflection sites and the periphery of the arterial system. There is a definite phase shift in time between the forward and the backward running components in a pulse pressure waveform. A normal wrist pulse waveform has a smooth, fairly sharp upstroke, a momentarily sustained peak, a quick down-stroke and decay. The reflected wave also has similar shape to the initial wave but smaller in amplitude. Figure 1 shows the standard pulse from the radial artery.

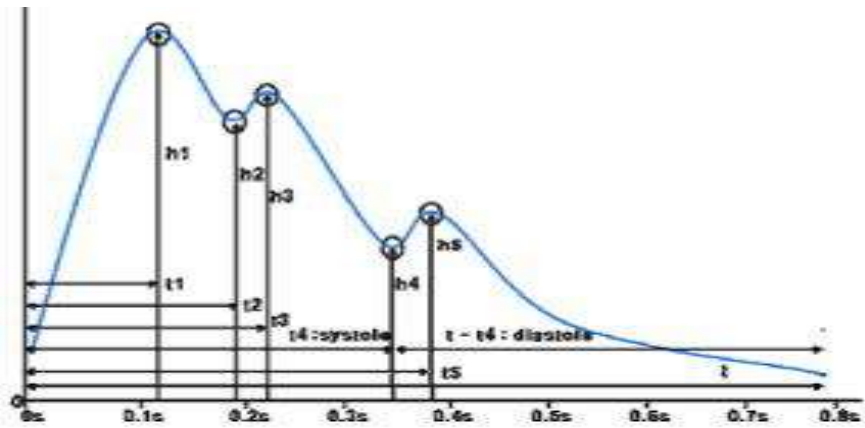

Fig. 1 Standard pulse pressure signal

The radial pulse wave has three peaks and five extreme a in general. $\mathrm{h} 1$ of first peak determines systole which reflects the blood ejaculation by heart contraction and $\mathrm{h} 3 \mathrm{~h} 5$ of second and third peak are related to the reflection sites to the down-ward travelling arterial pulse in the region of renal arteries and beyond the bifurcation of the iliac arteries. $\mathrm{t} 4$, which means the time of $\mathrm{h} 4$, is the start point of diastole.

\section{Limitations Of Indirect Measurement Of Arterial}

\section{Blood Pressure}

The theory of non-invasive measurement of arterial pressure is robust (Geddes 1970, Webster1997). It predicts that features of the K-sounds or the oscillogram are closely associated with specific levels of cuff pressure which are related to intra-arterial pressure. However, since it is the cuff pressure which is actually measured, the associations are dependent on the transduction process, whose fidelity cannot be readily determined. It is assumed that if all the correct steps are taken, the cuff pressure is a reliable measurement of arterial pressure. However, there is a general tendency for cuff pressure to underestimate true arterial systolic pressure independent of device (Borow and Newburger 1982, Hunyor et al 1978) with increased variability during exercise (Gould et al 1985). Although an association is found between cuff pressure and intraarterial pressure under optimum measurement conditions (Breit and O'Rourke 1974, O'Brien et al 2002, Ochiai et al 1997), the relation between peripheral blood pressure and central aortic pressure as a measure of left ventricular load is subject to a range of physiological variations. Figure 2 shows the correspondence between invasively measured radial artery pressure and aortic pressure (Pauca et al 1992). This study in adult subjects (age 48-77 years) showed a mean difference in the systolic pressure of $12 \mathrm{mmHg}$ (range -7 to 31 ), in the diastolic pressure of $-1.0 \mathrm{mmHg}$ (range -5 to 3 ) and in the mean pressure of $-0.8 \mathrm{mmHg}$ (range -5 to 3 ). The large scatter in systolic pressure results in a wide variation in pulse pressure. In addition to the methodological aspects of obtaining accurate measurement of pressures to correspond with intra-arterial pressure, the physiological phenomenon of the variable difference between central aortic and peripheral systolic pressure (figure 2) presents a potential limitation in using the conventional cuff sphygmomanometric pressure as a surrogate measure of left ventricular load, and as a fundamental descriptor of cardiovascular risk (McEniery et al 2008). 


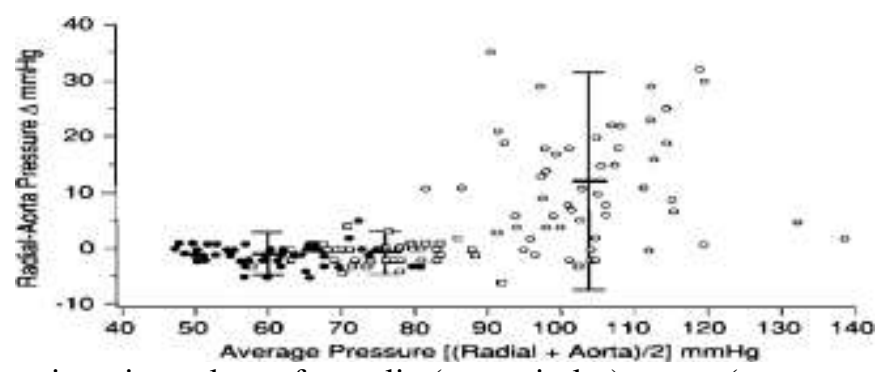

Fig. 2 Differences between invasive values of systolic (open circles), mean (open squares) and diastolic (closed circles) pressure between direct intra-arterial measurements of blood pressure, plotted against the average of the two pressures. Bars represent 2 standard deviations. The mean difference for mean and diastolic pressures is around zero, whereas the mean difference for systolic pressure is $12 \mathrm{mmHg}$, with a large scatter. Pauca et al (1992), with permission

\section{A. Characteristics of the cuff-pulse waveforms}

Waveforms acquired from blood pressure cuffs exhibit characteristics that are similar to, but not the same as arterial waveforms acquired by other methods. Even waveforms acquired simultaneously, but from different anatomical sites are not identical. The brachial cuff and wrist cuff waveforms in Fig. 4 illustrate this assertion. The top trace shows the wrist waveforms (WW) and the bottom trace shows arm (brachial) waveforms (AW) acquired simultaneously with the dual cuff system from an adult volunteer in the sitting position. The waveforms were acquired at the cuff pressure (CP) just below the point of DBP. The wrist waveforms have more sharply defined contours when compared with the brachial waveforms. The dicrotic notches on the descending part of the waveforms are well defined on the wrist waveforms. The brachial waveforms are more rounded and the dicrotic notches are barely visible. We believe that larger volume of air in the brachial cuff and larger amount of soft tissue on the upper arm cause the substantial damping of brachial cuff waveforms. Smaller volume of air and relatively low amount of soft tissue make the wrist cuff waveforms better suited for waveform analysis. It is important to acquire the waveforms at CP lower than the point of DBP. The waveforms shown in Fig 3 illustrate the need for appropriate cuff pressure. The waveforms were acquired during a gradual cuff deflation as is done during automatic BP measurement. The waveforms at cuff pressures above DBP are distorted because the radial artery is fully or partially occluded by the wrist cuff and blood flow under the cuff is turbulent. Turbulent blood flow is the source of Korotkoff sounds that are used in manual BP determination. When CP is lowered to pressures equal to or below DBP, the artery is no longer occluded, the waveforms are not distorted and Korotkoff sounds are no longer heard.

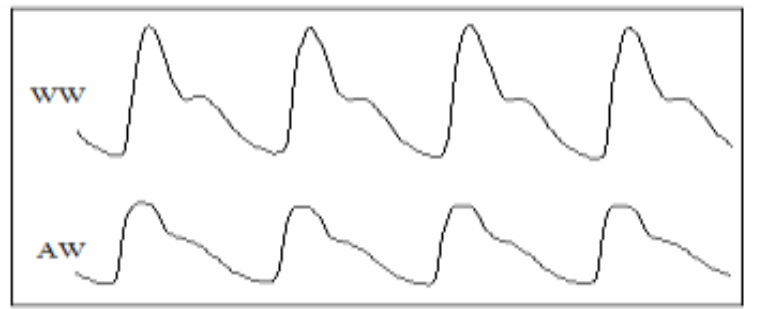

Fig. 3 Wrist waveforms (WW) and arm waveforms (AW) were acquired simultaneously.

\section{B. Preprocessing}

The data was filtered using a butterworth low pass filer of order 8 (cutoff - $7 \mathrm{~Hz}$ ) to remove any high frequency noise that might be present in the signal. The very low frequency components contained in a signal are sometimes an artifact caused either by the instruments used to acquire the signal or the movement of the subject, which shifts the PPG signal up or down. The PPG sensor is also very sensitive to these shifts. These low frequency components smear the power spectrum of the PPG signal and can affect the results. Furthermore, the PPG signal consists of a quasi DC signal that corresponds to changes in the venous pressure. While it is the arterial blood volume changes that have a direct bearing on cardiovascular dysfunction. This quasi DC signal needs to be removed and the signal was detrended before being subjected to analysis. A linear detrending was applied to the PPG data.

\section{C.Piezoelectric Film Properties:}

Piezo film is a flexible, lightweight, tough engineering plastic available in a wide variety of thicknesses and large areas. Its properties as a transducer include: 
- Wide frequency range $-0.001 \mathrm{~Hz}$ to $109 \mathrm{~Hz}$.

- Vast dynamic range (10-8 to $106 \mathrm{psi}$ or $\mu$ torr to Mbar).

- Low acoustic impedance — close match to water, human tissue and adhesive systems.

- High elastic compliance

- High voltage output-10 times higher than piezo ceramics for the same force input.

- High dielectric strength - withstanding strong Fields $(75 \mathrm{~V} / \mu \mathrm{m})$ where most piezo ceramics depolarize.

- High mechanical strength and impact resistance (109-1010 Pascal modulus).

- High stability—resisting moisture $(<0.02 \%$ moisture absorption), most chemicals, oxidants, and intense ultraviolet and nuclear radiation.

- Can be fabricated into unusual designs.

- Can be glued with commercial adhesives.

\section{Electronic PCB Design}

In order to acquire pulse pressure waveforms from human radial artery of the subject, the point on the wrist where best palpations of pulses were felt is marked with a marker. Then the sensor was placed on that point with help of finger. The sensor is given an external counter pressure with the spring mechanism stitched on to the strap band. Raw signals acquired from the sensor have very low amplitude i.e. in order of $0.01-5 \mathrm{mV}$. Hence, in order to observe and obtain the required characteristics of the waveform, it is required to condition and process the waveforms. For this, the acquired signals are first preamplified, then filtered and then amplified with higher gain. Figure 4 shows the block diagram of the instrumentation for acquiring the human radial arterial pulse pressure waveform.

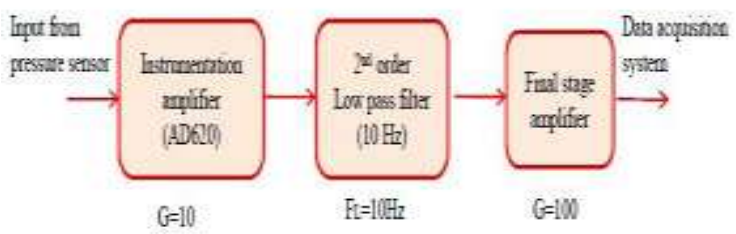

Fig. 4 Block Diagram of Signal Processing circuit

Pre-amplifier : stage boosts up the amplitude of the acquired signal. This stage was constructed using an instrumentation amplifier IC AD620 with the gain of 10.The advantage of employing this amplifier was that its CMRR is quite high so that the noise acquired from the external environment would be reduced.

Low pass Filter: (2nd order Butterworth Filter) with a cut off frequency of $10 \mathrm{~Hz}$ is employed to remove high frequency components and to get a clean pulse pressure waveform.

Final stage amplifier: was employed using a non-inverting amplifier with a gain of 100, using IC LM324.This stage was mainly employed to reduce the loading effect. Lowpass filter stage and final amplifier stage was realized using a quad Op-amp IC LM324. Figure 5 shows the signal processing circuit to be employed with sensor.

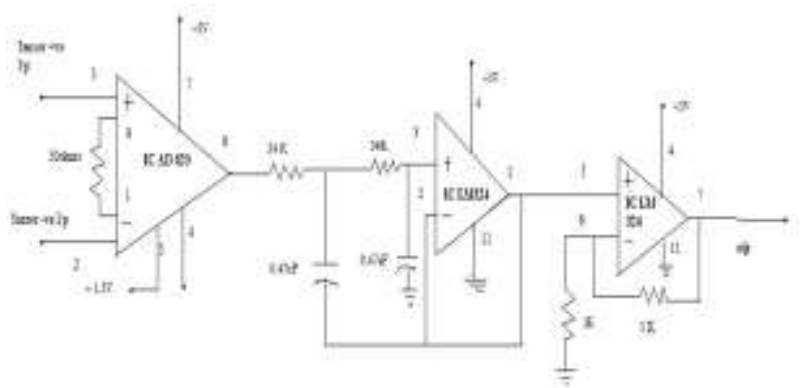

Fig. 5 Circuit Diagram for Signal Processing circuit 


\section{Basic Physiology}

Arterial blood pressure is generated by the ejection of blood from the left ventricle (LV) into the arterial vasculature. The amount of pressure generated is determined by the volume of blood ejected as well as the resistance to ejection within the systemic vascular network. In other words:

\section{PRESSURE $=$ Flow $X$ Resistance}

As blood is ejected into the proximal portion of the aorta, the vessel wall stretches and distends to accommodate the increase in volume. This stretching of the aortic segment is transmitted peripherally along adjacent segments of the aorta, thus producing a pulse wave that is transmitted through the arterial circulation and felt peripherally as a pulse.

The rate at which the pulse travels down the aorta is determined by the compliance and distensibility (vasodilation) of the arterial system. Decreases in compliance (hypertension or vasoconstriction) results in a rapid transmission of pulse wave. As the pulse travels peripherally from the central aorta, it changes shape as well as value. This occurs as a result of the reflected waves that summate on the primary systolic wave. The arterial pressure wave is divided into two phases that correspond to the cardiac cycle: systole and diastole. Arterial systole begins with the opening of the aortic valve and the rapid ejection of blood into the aorta. This is followed by "run-off" of blood from the proximal aorta to the peripheral arteries. On the arterial pressure waveform, this appears as a sharp rise in pressure followed by a decline in pressure. As pressure falls, the aortic valve snaps shut, causing a small change in arterial pressure that appears as a dip on the down slope. This is termed the "incisure" in central aortic pressures and the "dicrotic notch" in peripheral pressures. This marks the end of the ejection period. The "peak systolic pressure" is a reflection of maximum pressure generated by the LV and is normally $100-140 \mathrm{mmHg}$. Systolic pressure is frequently used to monitor ventricular "afterload". Afterload is defined as LV wall tension during systole. The two principal determinants are systolic pressure and the radius of the left ventricle (related to LV volume) is assumed to remain relatively constant, and therefore systolic pressure is the parameter used to clinically monitor after loads. Diastole follows closure of the aortic valve and continues until next systole. During this time, "run-off" to the peripheral arteries occurs without further flow from the LV. On the peripheral arterial waveform this is seen as a gradual decrease in pressure. The lowest point on diastole (end-diastole) is referred to as arterial diastolic pressure and is normally $60-80 \mathrm{~mm} \mathrm{Hg}$. The diastolic arterial blood pressure reflects both the velocity of "run-off" and the elasticity of the arterial system. The elastic properties of the vessels affects the arterial system's ability to change luminal dimensions as blood volumes change. Heart rate also affects the diastolic pressure because it determines the duration of diastole in the cardiac cycle. The longer the period of time and the shorter the diastolic period (with tachycardia), the higher the diastolic pressure. Diastolic pressure is also important in determining coronary perfusion, especially to the left ventricle, where most flow occurs during diastole. Normal coronary artery perfusion pressure is 60 to $80 \mathrm{~mm} \mathrm{Hg}$. Perfusion pressures below $50 \mathrm{mmHg}$ threaten myocardial perfusion. The arterial pressure differs in both contour and value depending on location of the artery. The systolic pressure tends to be higher in the femoral artery than in the radial or brachial artery by as much as 25 to $50 \mathrm{mmHg}$. Generally, the diastolic and mean arterial pressure (MAP) remains nearly the same. Normal arterial blood pressure is approximately $120 / 80 \mathrm{mmHg}$ in the healthy dog and cat. Systolic pressure greater than $180 \mathrm{mmHg}$ and diastolic pressures greater than $120 \mathrm{mmHg}$ are suggestive of hypertension. MAP is defined as the sum of diastolic pressure plus one third of the pulse pressure. It can also be mathematically calculated as:

\section{MAP $=\frac{\text { Systolic pressure }+(\text { Diastolic pressure X 2) }}{3}$}

MAP represents the average pressure within the arterial system. Since diastole typically lasts approximately two thirds of the entire cardiac cycle, the mean arterial pressure value is closer to diastolic value than to the systolic value. Even though, the arterial pressure pulse is amplified as it travel away from the heart, the MAP remains relatively unchanged, thus its importance as a reflection of overall perfusion. Normal MAP is 70-90 mm Hg. MAP dependent on two factors:

1. Cardiac Output (CO): The blood flow through the vessel.

. 2.Systemic Vascular Resistance (SVR): The elasticity of resistance of the vessels.

The above interrelationship can be expressed as:

$\mathrm{MAP}=$ Cardiac output $\mathrm{X}$ Systemic vascular resistance.

The pulse is the difference between the systolic and diastolic pressure. In other words:

Pulse Pressure $=$ Systolic Pressure - Diastolic pressure

The pulse pressure is largely determined by stroke volume (amount of blood ejected with each contraction), the compliance or distensibility of the arterial network and the character of ejection from the heart during systole. Thus, it is said that pulse pressure is reflective of stroke volume and arterial compliance. Any factor that increases stroke volume, such as decrease in pulse rate or an increase in $\mathrm{CO}$, also increases pulse pressure. A decrease in compliance or distensibility of the arterial network also tends to increase pulse pressure. In the emergency and critically ill patient, wide pulse pressures are associated with large stroke volumes, and 
narrow pulse pressures are seen with low stroke volume. The pulse pressure and not the systolic blood pressure largely determine the strength of a pulse on palpation. A weak peripheral pulse is generally associated with a stroke volume that is lower than normal. This could be due to a rapid pulse rate or decreased cardiac output. However, it is important to remember that, weak peripheral pulses at the extremities can also be due to arteries and arterioles severely constricted. Pulse pressures changes reflect alterations in cardiac output much more accurately than systolic blood pressure as shock develops. In hypovolemic shock, major decreases in stroke volume and pulse pressure as often occur long before there is significant fall in the systolic pressure. Diastolic pressure often rises initially with hemorrhage due to sympathadrenal stimulation, particularly if there is pain or tissue damage. Since vasoconstriction can increase only to a certain maximum, continued blood loss will eventually result in a fall in both systolic and diastolic pressures.

Therefore, in an individual patient, changes in pulse pressure can often correlate quite well with changes in stroke volume. An example of this can be of a patient's blood pressure changing from 120/80 to 110/90. In this particular case, the pulse pressure has fallen from $40 \mathrm{~mm} \mathrm{Hg}$ to $20 \mathrm{~mm} \mathrm{Hg}$. A $50 \%$ decrease in pulse pressure, could also signify a $50 \%$ decrease in stroke volume.

\section{Results And Discussion:}

Pulse pressure waveform varies with subjects based on their age, gender and cardiovascular functioning. To optimize the amplitude of pulse pressure waveform, a constant external pressure has to be applied based on wearer's physiology. A mechanism capable of fixing the optimal pressure waves acquired from the pressure sensor to optimally derive the systolic and diastolic values.

\section{Conclusion:}

Radial artery is the best site to acquire pulse pressure waveform, mainly over the anatomical snuff box on the wrist, for this reason it had been employed since ages for diagnosis of diseases. The characteristic Pulse Pressure waveforms obtained contains many features which depict the cardio vascular conditions of the subject. Thus if some methods are developed to study these waves in an objective manner, then these waveforms could replace many invasive diagnostic methods. Pulse pressure, which is a very important physiological parameter, could be calculated very accurately from these waveforms. The Pulse pressure waveforms obtained using miniature pressure sensor are exact representation of the standard pulse pressure, and by using these waveform patterns,pulse pressure could be calculated accurately. The modern world requires a state-of-the-art wearable pulse pressure monitoring system to overcome the increasing myocardial infarctions and other cardiovascular non-linearity. The developed algorithm described in this study has to be made robust by efficiently detecting all the morphological features of pulse pressure waveform during person's normal physical activities which generates non linear motion artifacts

\section{References}

[1]. Arthur C. Guyton and John E.Hall "Textbook of medical physiology",10th edition, pp.144-223, 2000.

[2] Bhaskar Thakker, Anoop Lal Vyas, and D M Tripathi, "Radial Pulse Analysis at Deep Pressure in Abnormal Health Conditions", IEEE, 3rdInternational Conference on Biomedical Engineering and Informatics October 2010.

[3] Chu-Chang Tyan, Shing-Hong Liu, Jan-Yow Chen, Jian-Jung Chen, and Wen-Miin Liang," A Novel Noninvasive Measurement Technique forAnalyzing the Pressure Pulse Waveform of the Radial Artery" IEEE Transactions on Biomedical Engineering, Vol: 55 , No: 1, January 2008

[4] George oliver,M.D,Lond,F.R.C.P. "Pulse gauging"- a clinical study of radial measurement and pulse pressure and studies in blood pressure, $3^{\text {rd }}$ edition,pp.72-87,2003.

[5] Jibing Gong, Shilong Lu, Rui Wang (Member IEEE), Li Cui (Member IEEE), "Low-cost and Wearable Healthcare Monitoring Systemfor Pulse Analysis in Traditional Chinese Medicine", IEEE, pp.205-227, April 2010.

[6] Jay N. Cohn, Stanley Finkelstein, Gary McVeigh, Dennis Morgan, Lisa LeMay, Jennifer Robinson, and James Mock, "Noninvasive Pulse Wave Analysis for the Early Detection of Vascular Disease, Hypertension", 26: 503-508. 1995.

[7] Willis J.Tompkins "Biomedical Digital Signal Processing", pp.220-260,1993.

[8] B.Thakker and A. L. Vyas, "Outlier Pulse Detection and Feature Extraction for Wrist Pulse Analysis", Proc. of ICBST'09, pp. 173176,2009 .

[9] Qun Wang, Zhiwen Liu, "The Pulse Waveform Characteristic Points Detection Based on Wavelet Transform", IEEE, 1st International Conference on Information Science and Engineering (ICISE2009). 